\title{
Extending Formal Reasoning with Support for Hardware Diagrams
}

\author{
Kathi Fisler \\ Department of Computer Science \\ Lindley Hall 215 \\ Indiana. University \\ Bloomington, IN 47405 \\ kfisler@cs.indiana.edu
}

\begin{abstract}
Diagrams have been left as an informal tool in hardware reasoning, thus rendering them unacceptable representations within formal reasoning systems. We demonstrate some advantages of formally supporting diagrams in hardware verification systems via a simple example from the verification of a single-pulser.
\end{abstract}

\section{Introduction}

Diagrams have been treated as second-class citizens within the realm of formal reasoning, despite their steady use as informal design tools. The reasons for this appear to be based more on prejudice against diagrams in logic rather than on any inherent properties that render diagrams inappropriate for formal use. Diagrams offer several potential advantages to hardware reasoning: they offer clear, compact and user-transferable representations, and they lack the high learning overhead associated with the formal logics underlying many state-of-the-art sentential reasoning tools. In fact, it would seem that the only thing precluding the rigorous use of diagrams in formal verification is the lack of formalization of diagrammatic representations.

This paper presents initial work in a research project aimed at exploring the interactions of diagrams and sentential representations in the context of hardware design and verification. The goals of this research project are to develop a heterogeneous logic of interacting sentential and diagrammatic representations and to build a proof-checker based upon the logic. Our logic supports four representations: timing diagrams, circuit diagrams, algorithmic state machine (ASM) charts, and higher-order logic. Rules of inference bridge representations, allowing all four representations to interact during the proof process.

\section{Previous Work}

Using visual representations in hardware design frameworks is not a new idea. Various design tools and description languages have employed diagrammatic representations [5] [7] [13], and systems for reasoning about some aspects of systems 
using diagrammatic representations have appeared over the past year [3] [2] [10] [12]. Many systems provide formalizations of timing diagrams [2] [10] [12] and some even provide formal definitions of the interaction between timing diagrams and sentential representations [12]; however, none of these support multiple diagrammatic representations. The authors of [3] present a system in which a user can reason about system states using a graphical interval logic, but they translate their visual representations into a sentential logic for purposes of formal manipulation, unlike our logic, which is developed directly at the level of the diagrams. A previous attempt at defining a heterogeneous logic for hardware, along with more complete arguments supporting the use of diagrams in hardware formal methods, is presented in [8]. The logic presented in [8] is less fine-grained than the one presented here; their logic is based only on behavioral relationships while this work allows for reasoning about structural relationships between components. To the best of our knowledge, this work is the first to present examples of fully formal reasoning using diagrammatic logic in the hardware and formal methods communities.

The motivation for our research is based largely upon Hyperproof, the heterogeneous logic reasoning tool developed by Barwise and Etchemendy [1]. Hyperproof consists of a proof-checker for a logic of sentential and diagrammatic representations in a blocks world. We envision constructing an initial tool with much of the same flavor as Hyperproof.

\section{Verification and Diagrammatic Representation}

We believe that diagrammatic reasoning offers two main advantages in hardware verification: clarity of representation and conciseness of proof. We will use the single-pulser to demonstrate our arguments. The single-pulser is a good choice for this due to its size and clarity; such a study also complements the studies of single-pulser verifications given in these proceedings [9]. We use the work of [9] in this discussion as representative of sentential verification efforts.

To address the issue of clarity, consider the PVS implementation and specification of the single-pulser proposed by [9]. Their implementation is given below and is based upon the accompanying circuit diagram.

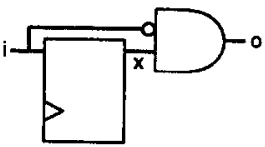

$$
\begin{aligned}
& \operatorname{imp}(i, O): \text { bool }=\left(\exists x:\left(\operatorname{delay}(i, x) \wedge \operatorname{and}_{0}^{\circ}(i, x, O)\right)\right) \\
& \operatorname{delay}(i, O): \text { bool }=(\forall t:(O(t+1)=i(t))) \\
& \operatorname{and}_{\circ}^{\circ}(a, b, c): \text { bool }=(\forall t:(c(t)=(-a(t)) \times b(t)))
\end{aligned}
$$

Notice that the PVS representation is nothing but a translation of the diagram into the syntax of PVS - neither representation contains any more information than the other. ${ }^{1}$ The behavioral specification of [9] is given in two parts,
as follows:

${ }^{1}$ We could consider the lengths of wires in the circuit diagram as information not available in the sentential representation, but that information is more fine-grained than our logic is tuned to handle at present. 


$$
\begin{aligned}
& \operatorname{spec} 1(i, O): \text { bool }=(\forall n, m: \operatorname{Pulse}(i, n, m) \supset \\
& \exists k: n \leq k \wedge k \leq m \wedge O(k)=1 \wedge \\
& (\forall j:(n \leq j \wedge j \leq m \wedge O(j)=1 \supset j=k)) \\
& \operatorname{spec} 2(i, O): \text { bool }=(\forall k: O(k)=1 \supset \text { SinglePulse }(O, k) \wedge \\
& (\exists n, m: n \leq k \wedge k \leq m \wedge P \text { ulse }(i, n, m)))
\end{aligned}
$$

The same specification could be given in terms of the following:

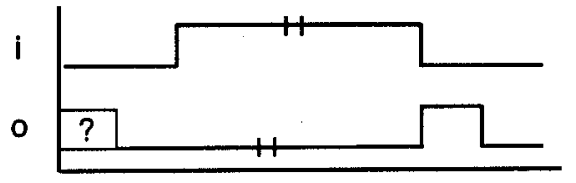

We claim that the timing diagram is a clearer representation of the intended behavior of a single-pulser than the two sentential specifications. The meaning of neither sentential specification is immediately clear, despite the fact that they are written in a straightforward style of higher-order logic. In fact, the average person might construct a diagrammatic depiction of the specifications in the process of understanding their full meanings. The advantages of clear specification are well known in the verification community. Careless interpretation of either specifications or implementations can lead to lost time in establishing proofs, or worse still, invalid proofs of correctness. Of course, the argument can also be made that there are issues of interpretation involved in using diagrams as well; we agree, but claim that the clarity of properly formalized diagrammatic representations minimizes the problem.

We now turn to comparing the sentential single-pulser verification to a possible diagrammatic verification. There are two aspects to consider: the time to develop proofs and the conciseness of the resulting proof. The PVS proof referenced in [9] took an estimated half-hour of proof time for a relatively novice PVS user; the main time expenditure was in properly formulating the specification, which took considerably longer than the actual verification [11]. Although we have no evidence to support this, we believe that specifications may be easier to state and debug using diagrammatic representations that are more familiar to practicing designers.

A brief discussion of our rules of inference is in order; our intent is to design the logic such that diagrammatic rules of inference mimic the informal reasoning steps used by designers in practice. The inference rules relating and gates and timing diagrams appear below; other inference rules on and gates, such as one where a low input yields a low output, can be derived from these three primitive rules. The portion of the logic necessary to support these rules can be found in [6]. 

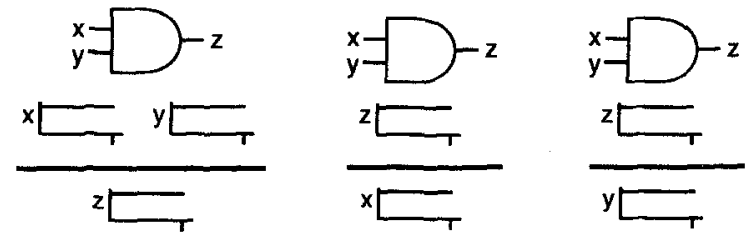

Now consider the following diagrammatic proof that corresponds to the proof of spec1. In the timing diagrams, the dashed axis notation denotes that the dashed tick repeats the number of times indicated on the dashed line.

1.

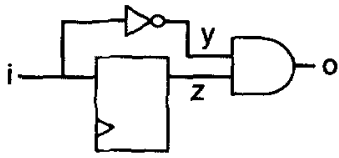

2.

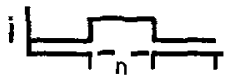

3.

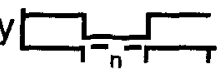

4.

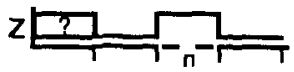

5. a. $n=1$

b.

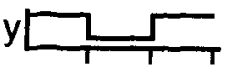

c.

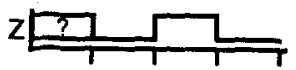

d.

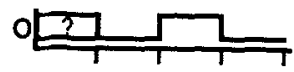

6. a. $n>1$

b.

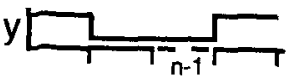

c.

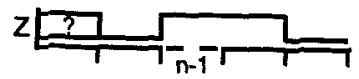

d.

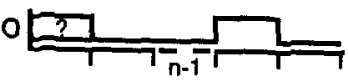

7.

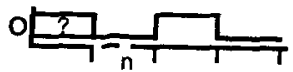

Assume

Assume

Inverter Rule, 2

Unit Delay Rule, 2

Assume

Instantiate value of $n, 3$

Instantiate value of $n, 4$

And Rule, 5b, 5c

Assume

Repetition expansion, 3

Repetition expansion, 4

And Rule, 6b, 6c

Merge, 5d, 6d

Comparing this proof to the PVS proof trace given in [9], it seems reasonable to argue that the diagrammatic proof is easier to follow and quite possibly easier to produce than the one required to verify spec1 in PVS. The steps taken in the 
diagrammatic proof are also at a lower granularity (for sake of example) than those we expect to be taken in practice, thus compacting the proof even further. Though we have no measured results to support this, empirical evidence using Hyperproof indicates that proofs are often substantially shorter than their purely sentential equivalents [4].

The above presentation argues the benefits of using diagrammatic representations in formal verification, but it does not adequately address our particular approach of developing a logic of hardware diagrams. Certainly it would seem reasonable to merely provide a diagrammatic interface to an existing sentential logic, thereby allowing us to rely on existing tools for verification. There are certain obvious immediate benefits to such an approach, such as the timeliness with which diagrams could be used to aid in formal verification.

We believe, however, that this approach is not the correct one to take for three reasons. By using diagrams merely as an interface tool, we leave them as secondclass citizens to sentential logic in the realm of formal reasoning. We believe that diagrams are as valid a representation as sentential forms in reasoning and we are interested in the creation of logics that put diagrams on equal par as a valid representation. In addition, using diagrams merely in an interface capacity sidesteps our belief that there are logical relationships between different diagrammatic hardware representations. Identifying these relationships may lead us to even stronger frameworks for verification, but to do so requires closer examination of the diagrams themselves as first-class citizens.

Finally, we are not convinced that translation is a desirable approach. Any formal system that uses translation needs to prove that the translation is done correctly; such an argument will require some level of proof that operates on the diagrams, so translation does not save us from needing to formally consider diagrams in proof. In addition, manipulations might be made on the translated representation that do not naturally translate back up to the diagrammatic representation; this problem would be critical for a system that implements inferences on diagrams. Lastly, there may exist natural rules of inference on diagrams that become less natural, perhaps even unwieidy, at the sentential level; this seems at odds with our goal of providing natural diagrammatic inferences.

\section{Conclusions and Future Work}

We have presented a simple example demonstrating that diagrammatic representations can be formalized and used effectively in proof. Although the actual logic is not presented here for sake of space, the example proof is correct within the logic we have developed, and all inferences used in the example proof have been proven sound. A more complete discussion of the proof and a presentation of the logic that supports it is provided in [6].

We are in the process of completing the definition of the logic presented here; this includes presenting rules of inference and establishing soundness and completeness results for the full logic. The current status of this work is available in [6]. A prototype implementation of a simple proof-checker based upon our 
logic is in an early development stage. We have yet to apply the logic to a substantial verification effort, but plan to do so in the near future. What is presented here is merely an initial attempt to formalize the interactions between these various diagrammatic representations. There is still much research to do both in understanding the role of diagrams in verification and in developing tools that use such formalizations.

\section{Acknowledgements}

The author would like to thank Jon Barwise, Steve Johnson, Gerry Allwein, and Shriram Krishnamurthi for their helpful comments in both the research and the preparation of this paper.

\section{References}

1. Jon Barwise and John Etchemendy. Hyperproof, CSLI Lecture Notes, University of Chicago Press. To appear, 1994.

2. Viktor Cingel. A graph-based method for timing diagrams representation and verification. In George J. Milne and Laurence Pierre, editors, Correct Hardware Design and Verification Methods, pages 1-14. CHARME, Springer-Verlag, 1993.

3. L.K. Dillon, G. Kutty, L.E. Moser, P.M. Melliar-Smith, and Y.S. Ramakrishna. A graphical interval logic for specifying concurrent systems. Technical report, UCSB, 1993.

4. Ruth Eberle, April 1994. Personal communication.

5. Simon Finn, Michael P. Fourman, Michael Francis, and Robert Harris. Formal system design - interactive synthesis based on computer-assisted formal reasoning. In Luc Claesen, editor, Formal VLSI Specification and Synthesis: VLSI DesignMethods-I. North-Holland, 1990.

6. Kathi Fisler. A logical formalization of hardware design diagrams. Indiana University Technical Report TR416, September 1994.

7. Graham Hutton. The Ruby interpreter. Technical Report 72, Chalmers University of Technology, May 1993.

8. Steven D. Johnson, Gerard Allwein, and Jon Barwise. Toward the rigorous use of diagrams in reasoning about hardware. IULG Preprint Series, April 1993.

9. Steven D. Johnson, Paul Miner, and Shyam Pullela. Studies of the single-pulser in various reasoning systems. In Theorem-Provers and Circuit Design Proceedings, September 1994.

10. K. Khordoc, M. Dufresne, E. Cerny, P.A. Babkine, and A. Silburt. Integrating behavior and timing in executable specifications. In $C H D L$, pages 385-402, April 1993.

11. Paul S. Miner, July 1994. Personal communication.

12. Rainer Schlör and Werner Damm. Specification and verification of system-level hardware designs using timing diagrams. In Proc. European Conf. on Design and Automation, Paris, February 1993.

13. Mandayam Srivas and Mark Bickford. SPECTOOL: A computer-aided verification tool for hardware designs, vol I. Technical Report RL-TR-91-339, Rome Laboratory, Griffiss Air Force Base, NY, December 1991. 